\title{
Amplified Spontaneous Emission of Proton Transfer Dyes in Polymers
}

\author{
C. E. Fellows ${ }^{1}$, U. Täuber ${ }^{1}$, C. E. M. Carvalho ${ }^{2}$, and C. G. Carvalhaes ${ }^{3}$ \\ ${ }^{1}$ Laboratório de Espectroscopia e Laser, Instituto de Física, \\ Universidade Federal Fluminense, Av. Gal. Milton Tavares de Souza, s/n, \\ Campus da Praia Vermelha, Boa Viagem, Niterói, RJ, 24210-340, Brazil \\ ${ }^{2}$ Laboratório de Fotoquímica, Instituto de Química, Universidade Federal Fluminense, \\ Campus do Valonguinho, Centro, Niterói, RJ, 24020-150, Brazil and \\ ${ }^{3}$ Instituto de Matemática e Estatística, Universidade do Estado do Rio de Janeiro, \\ Rua São Francisco Xavier, 524, Maracanã, RJ, 20559-900, Brazil
}

Received on 7 January, 2005

\begin{abstract}
We compare the Amplified Spontaneous Emission (ASE) of the proton transfer dyes 2-(2'-hydroxyphenyl) benzimidazole (HPBI) and 2-(2'-hydroxy-5'-chlorphenyl)benzimidazole (Cl-HPBI) in three different polymeric hosts under UV pulsed laser excitation. Pulseform and degradation of the dyes in the solid polymers were analysed. Spectroscopic data were used in a simple four-level kinetic rate equation system, in the attempt to explain the observed results, i.e. first order exponential decay of maximum output energy and pulse shortening. The model includes transitions for the normal and tautomer form of the molecule as well as intersystem crossing and triplet-triplet transitions.
\end{abstract}

\section{INTRODUCTION}

Incorporation of dyes in solid materials instead of liquids is an idea that is as old as dye lasers itself [1]. From application point of view polymers are the first choice as solid host material due to the well-established production techniques and the very low price. A dye filled polymeric rod as active laser material could replace dye cell and circulating pump. In this way the extensive spectra of laser dyes can be used with the advantages of the solid-state lasers to be combined to an allsolid-state dye laser. However, polymers are poor thermal conductors and the degradation of host and dye material is high, because there is no refreshing process like thermal diffusion or circulating pumping in the case of liquid solvents. Dyes that undergo intramolecular proton transfer (IPT) in the excited state show a large Stokes shift and low self-absorption. That is why it was supposed that IPT dyes will show a reduced photo degradation. However, the degradation of IPT dyes dissolved in solid polymers is unexpected high [2,3]. Covalent bonding of the dye molecules to the polymer backbone via copolymerisation will reduce the photodestructive processes, [2]. In this way a notable increase of efficiency was realised. But compared with non proton-transfer dyes, like Coumarin and Rhodamine [4,5], the effect of copolymerisation to photo stability is minor. There was a lot of work done to find new dye-polymer complexes with reduced photo degradation, but no model is given in the literature that explains the photodestructive processes in IPT dyes and the effects to laser activity.

Amplified Spontaneous Emission (ASE) is the fundamental process in any laser activity. If the pumping beam form a narrow but long enough line in the active media the ASE result in a superradiant beam even without any laser cavity. In the present work the ASE properties of 2-(2'-hydroxyphenyl) benzimidazole (HPBI) and 2(2'-hydroxy-5' -chlorphenyl)benzimidazole (Cl-HPBI) incorporated in PMMA, PS and a PS/PMMA copolymer were investigated to estimate the influence of the polymer matrix on emission efficiency and photo stability of these dyes. Simu- lation of the ASE output pulse has also been performed. The rate equations and parameters used in these calculations are illustrated and a comparison with experimental results was done.

\section{SAMPLE PREPARATION AND SET UP}

2-(2'-hydroxyphenyl)benzimidazole (HPBI) and 2-(2'hydroxy-5' -chlorphenyl) benzimidazole (Cl-HPBI) were prepared by condensation of equimolar amounts of phenylene diamine with the adequate 2-hydroxybenzoic acid in phosphoric acid medium [6]. For spectroscopic investigations the dyes have been incorporated in polymers dissolved in chloroform followed by vaporisation of the chloroform resulting in films of 10 to $100 \mu \mathrm{m}$ thickness. Absorbance was measured with Carry 1E spectrometer, fluorescence with Hitachi F-4500 fluorescence spectrometer.

Quantum yield was calculated by comparison with the fluorescence spectrum of 9,10-diphenylanthracene of same optical density. Fluorescence lifetime was measured with Edinburgh Instruments DC-900 Time-resolved Spectrofluorimenter. Lifetimes have been measured in two different concentrations: $1.4 \times 10^{-3} \mathrm{M}$, used in the blocks for ASE experiments, and $1.7 \times 10^{-5} \mathrm{M}$. There are no significant differences in the lifetimes that indicate the absence of considerable intermolecular processes.

For ASE experiments polymer devices were prepared by the solubilization of the respective dye in monomer and the polymerisation started with benzoyl peroxyde moulded in a test tube of $1 \mathrm{~cm}$ diameter. The polymerisation process was executed at $50^{\circ} \mathrm{C}$ for 1 day. After polymerisation process the test tubes were broken and the originating rods of doped polymers (ca. $3.5 \times \phi 1 \mathrm{~cm}$ ) were cutted and polished manually on one end. The surface parallel to the axis of the rod was used without further preparation. The samples were transversely pumped by a home made $\mathrm{N}_{2}$ laser $(337 \mathrm{~nm}, 80 \mu \mathrm{J}, 3$ ns pulses [7]) at a repetition rate of $1 \mathrm{~Hz}$. The pump light was 
focused by a cylindrical quartz lens resulting in a pump area of ca. $0.5 \times 10 \mathrm{~mm}$. The dye ASE output beam was parallel to the rod axis and perpendicular to the pump beam. The pump laser and dye ASE pulse were measured with ITL 1850 Diode, Photek PMT140 Photomultiplier Tube and Tektronix 7104 oscilloscope and Oriel Neutral Density Filters. No laser cavity was used resulting in pure ASE. Maximum and pulsewidth (FWHM) of the output ASE pulses were analysed. Excitation was done until emission stopped completely.

\section{EXPERIMENTAL RESULTS}

HPBI and Cl-HPBI show an intramolecular proton transfer (IPT) in the excited state. Fig. 1 shows the energy level diagram. Fig. 2 shows the fluorescence spectrum of HPBI dissolved in Polymethylmethacrylate (PMMA), Polystyrene (PS) and a 1:1 PS/PMMA copolymer (excitation wavelength $337 \mathrm{~nm})$. The spectral bandwidth was $60( \pm 2) \mathrm{nm}$ independent of dye and host material. The maximum of fluorescence emission in PMMA and PS was shifted $4 \mathrm{~nm}$ (HPBI) and 5 $\mathrm{nm}$ (Cl-HPBI) in comparison with the copolymer. For both dyes the emission quantum yield in PS/PMMA copolymer is the major one followed by PS and PMMA. Table 1 shows the measured spectroscopic data. As shown by Ferrer et al. [2], the emission spectra of HPBI in three different environments (fluid dioxane solution, solid solution of PMMA and copolymerized with MMA) are virtually identical in wavelength maximum, indicating that there is no reaction between polymerization radicals and dye. Doping the preformed polymers with dyes has been tried but the blocks do not show optical quality for ASE experiments.

A summary of the results of ASE experiments is given in
Table 2. The maximum output energy was observed in the PS/PMMA co-polymer as host material with an initial efficiency of $6.5 \%$ for HPBI and $5.1 \%$ for Cl-HPBI. This corresponds to the observed emission quantum yields. Fig. 3 shows the relative ASE intensity in function of accumulated absorbed pump energy, i.e. energy per pump pulse multiplied by the number of pulses. In PS/PMMA co-polymer the photo degradation of HPBI is the slightest followed by PS and PMMA (Fig. 3a). The degradation follows a first order exponential decay. Costela et al. [3] report for different 2-(2'hydroxyphenyl) benzimidazoles degradation by pumping with 1 to $3 \mathrm{~mJ}$ pulses at $337 \mathrm{~nm}$. The decay curves observed by these authors are in contrast to our results and far away from first order exponential behaviour. This result was attributed to complex photo degradation processes, probably due to the high energy used in their pump pulse. The very low pump energy of $80 \mu \mathrm{J}$ used in our experiments could result in the occurrence of only one of these processes, what is assumed for the calculation done in this work. Fig. 4 shows the pulsewidth vs. accumulated pump energy. There is a pulse shortening for both dyes in PS/PMMA and pure PS matrix material. The results of Cl-HPBI are similar to those of HPBI, Fig. $3 \mathrm{~b}$ and Fig. 4b.

\section{CALCULATIONS}

In order to explain the observed behaviour we used a four level kinetic model including normal and tautomer form triplet states. The model was proposed first time by Chou et al. to calculate the gain spectra of proton-transfer fluorescence of 3hydroxyflavone [8]. A fourth order Runge-Kutta routine was used to do the numerical integration of the following system of differential equations:

$$
\begin{gathered}
\frac{d N_{0}}{d t}=k_{S_{0}^{\prime} S_{0}} N_{0}^{\prime}+k_{T S_{0}} N_{T}-\sigma_{O p} I_{p} N_{0} \\
\frac{d N_{1}}{d t}=\sigma_{O p} I_{p} N_{0}-k_{S^{\prime} S} N_{1}^{\prime} \\
\frac{d N_{T}}{d t}=k_{T T} N_{T}^{\prime}-k_{T S_{0}} N_{T} \\
\frac{d N_{0}^{\prime}}{d t}=\left(k_{S^{\prime} r}+k_{S^{\prime} i c}\right) N_{1}^{\prime}+\sigma_{S}^{\prime} I_{A S E} N_{1}^{\prime}+k_{T^{\prime} S_{0}^{\prime}} N_{T}^{\prime}-\sigma_{O p}^{\prime} I_{p} N_{0}^{\prime}-\sigma_{O L}^{\prime} I_{L} N_{0}^{\prime}-k_{S_{0}^{\prime} S_{o}} N^{\prime} 0 \\
\frac{d N_{1}^{\prime}}{d t}=k_{S S^{\prime}} N_{1}+\sigma_{O p}^{\prime} I_{p} N_{0}^{\prime}+\sigma_{O L}^{\prime} I_{A S E} N_{0}^{\prime}-\frac{1}{\tau^{\prime}} N_{1}^{\prime}-\sigma_{S}^{\prime} I_{A S E} N_{1}^{\prime} \\
\frac{d N_{T}^{\prime}}{d t}=k_{S^{\prime} T^{\prime}} N_{1}^{\prime}-k_{T^{\prime} T} N_{T}^{\prime}-k_{T^{\prime} S_{0}^{\prime}} N_{T}^{\prime}
\end{gathered}
$$


Table 1. Parameters of used dye-polymer complexes

\begin{tabular}{|c|c|c|c|c|c|}
\hline Dye & $\begin{array}{c}\text { Host } \\
\text { coefficient } \varepsilon\end{array}$ & $\begin{array}{c}\text { absorption } \\
\text { quantum } \\
{\left[10^{3} 1 \mathrm{~mol}^{-1} \mathrm{~cm}^{-1}\right]}\end{array}$ & $\begin{array}{c}\text { emission } \\
\text { fluorescence } \\
\text { yield }\end{array}$ & $\begin{array}{c}\text { maximum of } \\
\text { lifetime [ns] } \\
\text { emission [nm] }\end{array}$ & fluorescence \\
\hline HPBI & PMMA & 51.2 & 0.15 & 461 & 4.8 \\
& PS & 51.3 & 0.52 & 465 & 4.5 \\
& PS/PMMA & 43.3 & 0.62 & 461 & 4.1 \\
\hline CI-HPBI & PMMA & 39.7 & 0.26 & 467 & 4.9 \\
& PS & 53.8 & 0.28 & 472 & 4.5 \\
& PS/PMMA & 41.2 & 0.48 & 467 & 4.7 \\
\hline
\end{tabular}

normal form

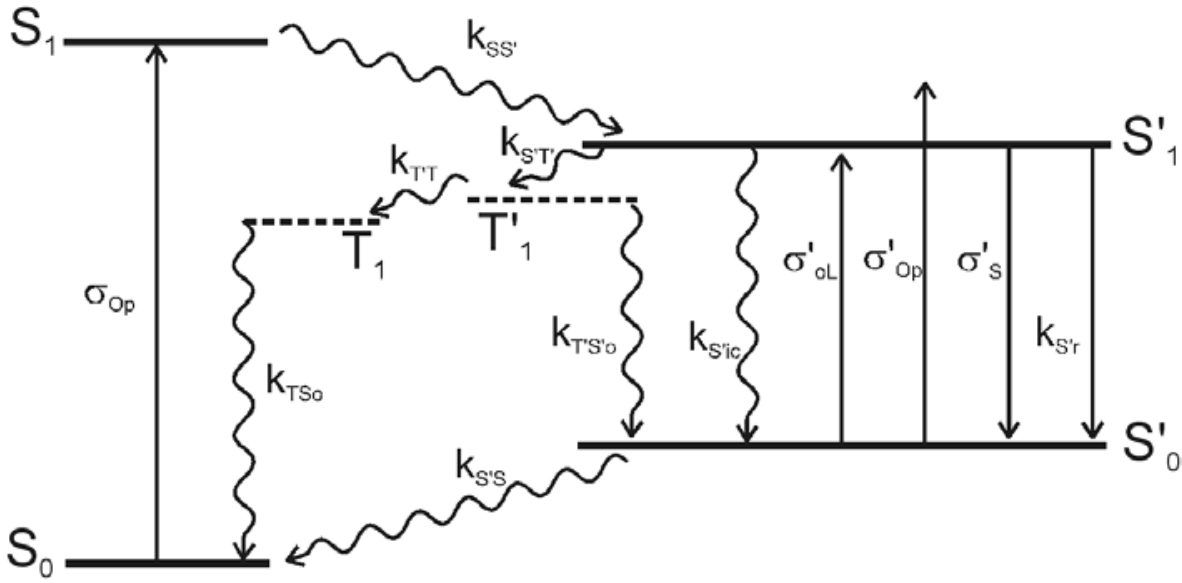

\footnotetext{
$k_{S_{0}^{\prime \prime} S_{0}}$ back proton within the singlet system $S_{0}^{\prime}-S_{0}$

intersystem crossing of the tautomer form $T$-So

$k_{T^{\prime} S_{0}^{\prime}}$ intersystem crossing of the normal form $T^{\prime}$-S'o

$k_{S S^{\prime}} \quad$ proton-transfer within the singlet system S-S'

$k_{T^{\prime} T} \quad$ back proton-transfer of the triplet system $T^{\prime}-T$

$k_{S^{\prime} r} \quad$ radiative desexcitation of $S^{\prime}$

$k_{S^{\prime} i c}$ non-radiative desexcitation of $S$

$k_{S^{\prime} T^{*}} \quad$ intersystem crossing of the tautomer form $S^{\prime}-T$,
}

$\sigma_{O p}$ absorption cross section of the normal form at pump

$\sigma^{\prime}{ }_{S}$ emission cross section of the tautomer form $\left(S_{1-}^{\prime} S_{0}^{\prime}\right)$

$\sigma_{O p}^{\prime}$ absorption cross section of the tautomer form at pump wavelength $\left(S_{0}-S_{1}\right)$

$\sigma{ }^{\prime} O L$ absorption cross section of the tautomer form at laser wavelength $\left(S_{0}-S_{1}\right)$

$\sigma_{1 T L} \quad$ triplet absorption cross section of the normal form at pump wavelength $\left(S_{0}-S_{1}\right)$

$\sigma^{\prime}{ }_{1 T L}$ triplet absorption cross section of the tautomer form at pump wavelength $\left(S_{0}-S_{1}\right)$

$\tau_{c}$ cavity decay time

$\phi_{F} \quad$ fluorescence quantum yield of
the tautomer form

FIG. 1: Energy diagram scheme and all relevant processes of IPT dyes. 
Table 2. Comparison of the results of the ASE experiments

\begin{tabular}{|c|c|c|c|c|c|}
\hline Dye & Host & $\begin{array}{c}\text { max.output } \\
\text { energy }[\mu \mathrm{J}]\end{array}$ & $\begin{array}{c}\text { max. } \\
\text { efficiency } \\
{[\%]}\end{array}$ & $\begin{array}{c}\text { 1/e decay of } \\
\text { output energy } \\
{[\mathrm{J}]}\end{array}$ & $\begin{array}{c}\text { max. } \\
\text { pulsewidth } \\
{[\mathrm{ns}]}\end{array}$ \\
\hline HPBI & PMMA & 2.74 & 3.4 & 1.98 & 2.08 \\
& PS & 1.27 & 1.6 & 7.08 & 2.30 \\
& PS/PMMA & 5.16 & 6.5 & 12.34 & 2.78 \\
\hline CI-HPBI & PMMA & 2.96 & 3.7 & 1.82 & 2.26 \\
& PS & 1.46 & 1.8 & 7.79 & 1.87 \\
& PS/PMMA & 4.06 & 5.1 & 9.11 & 2.26 \\
\hline
\end{tabular}

${ }^{a}$ Value of accumulated absorbed pump energy after a decay to 1/e of starting output energy based on first order exponential fit.

$$
\frac{d I_{A S E}}{d t}=\left(\frac{c I_{A S E}}{n}\right)\left(\sigma_{S}^{\prime} N_{1}^{\prime}-\sigma_{O L}^{\prime} N_{0}^{\prime}-\sigma_{1 T L}^{\prime} N_{T}^{\prime}-\sigma_{1 T L} N_{T}\right)-\left(\frac{I_{A S E}}{n \tau_{c}}\right)
$$

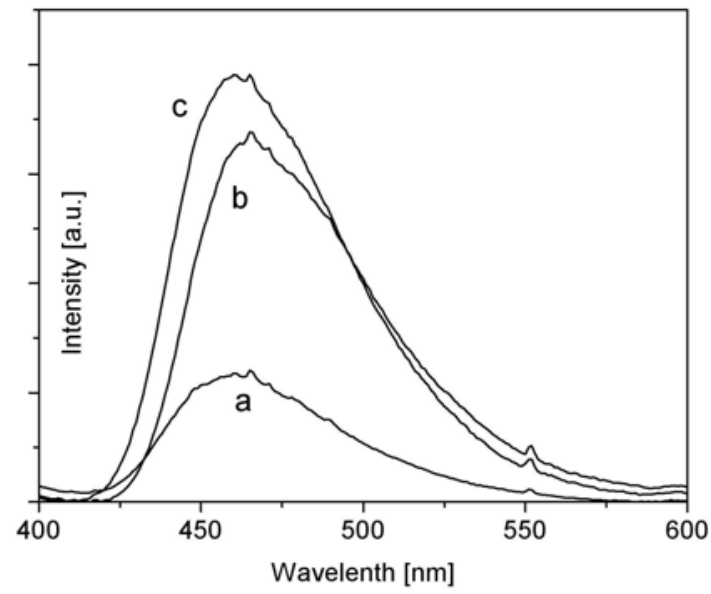

FIG. 2: Fluorescence spectrum of HPBI in (a) PMMA, (b) PS, (c) in PS/PMMA, excitation wavelength $337 \mathrm{~nm}$.

The $N_{i}$ are the population densities in molecules per $\mathrm{cm}^{3}$ in the the parameters, we used in the calculations are given elsewhere [9,10]. Fig. 5 shows the calculated ASE pulse (dotted line) of HPBI in PS/PMMA copolymer. The initial spike in the calculation is a typical spiking phenomenon due to the non-zero starting value of the laser intensity $I_{A S E}(t=0)$. The very rapid rise of $N_{I}^{\prime}$ with pump intensity leads to a marked population inversion between $S_{I}^{\prime}$ and $S_{0}^{\prime}$ and via the first two terms of (7) to a rise in laser intensity. To compare experimental results with calculations a digital $1 \sim \mathrm{GHz}$ low pass filter was used for smoothing the calculated curves (dashed line in Fig. 5).

\section{DISCUSSION}

The simulation was performed with several values of $N_{0}$ (numbers of molecules in the fundamental state $S_{0}$ per $\mathrm{cm}^{3}$ from 50 to $0.5 \times 10^{17}$ to investigate the behaviour of the model under conditions of degradation that courses a reduction of the number of active dye molecules. The dye concentration of the rods in the experiments was of $\sim 1.4 \times 10^{-3} \mathrm{M}$ what is equivalent to $8.43 \times 10^{17}$ molecules per $\mathrm{cm}^{3}$ and therefore on the lower region of the calculated range. The maximum of the output ASE pulse decreases linearly with the number of molecules $N_{0}$, Fig. 6 a. The internal proton transfer (IPT) from the excited normal form $\left(S_{1}\right)$ to the excited tautomer form $\left(S_{1}^{\prime}\right)$ is a very fast process with a time constant of some picoseconds $k_{S S^{\prime}}=10^{12} \mathrm{~s}^{-1}$ [10] what leads to a direct linkage of $N_{1}$ with $N_{1}^{\prime}$. Consequently all pumped molecules will immediately reach $N_{1}^{\prime}$ and contribute to the ASE process.

In contrast to the pulse maximum the pulsewidth of the output ASE pulse (FWHM) is nearly constant from $N_{0}=50$ to $10 \times 10^{17} \mathrm{~cm}^{-3}$ and decreases from 10 to $0.5 \times 10^{17}$, Fig. $6 \mathrm{~b}$. To explain this characteristic Fig. 7 shows the population densities of the fundamental tautomer state $N_{0}^{\prime}$, the normal triplet state $N_{T}$ and the intensity of the emitted ASE pulse for three different fundamental state concentrations $N_{0}$. There are two processes influencing the pulsewidth. Due to the first term of (2) a higher number of photons in the ground state $N_{0}$ leads to a faster decline of $N_{1}$ and through it the threshold of the amplification will be reached very fast. In Fig. 7 there is a $0.5 \mathrm{~ns}$ start delay between the calculated pulse of $N_{0}=50$ and $0.8 \times 10^{17} \mathrm{~cm}-3$. A second process causes a faster fall of the pulse for low concentrations. The population density $N^{\prime} 1$ of $S_{1}^{\prime}$ and consequently $N_{T}$ of $S_{T}$ goes non-linearly with $N_{0}$ because of the "self regulating" laser process $N_{0}^{\prime} \sigma_{S} I_{L}$ in equation (7). With decreasing $N_{0}$ the population of the metastable triplet states $N_{T}$ and $N_{T}^{\prime}$ increases in relation to $N_{0}^{\prime}$, and below $N_{0}=1 \times 10^{-17} \mathrm{~cm}^{-3}$ there is a noticeable absence of deactivated molecules in $S_{0}$ for restarting the excitation process within the excitation pulse. Repeating the calculations by exclusion of the laser process $\left(\sigma_{S}=0\right)$ keeps the relative pop- 

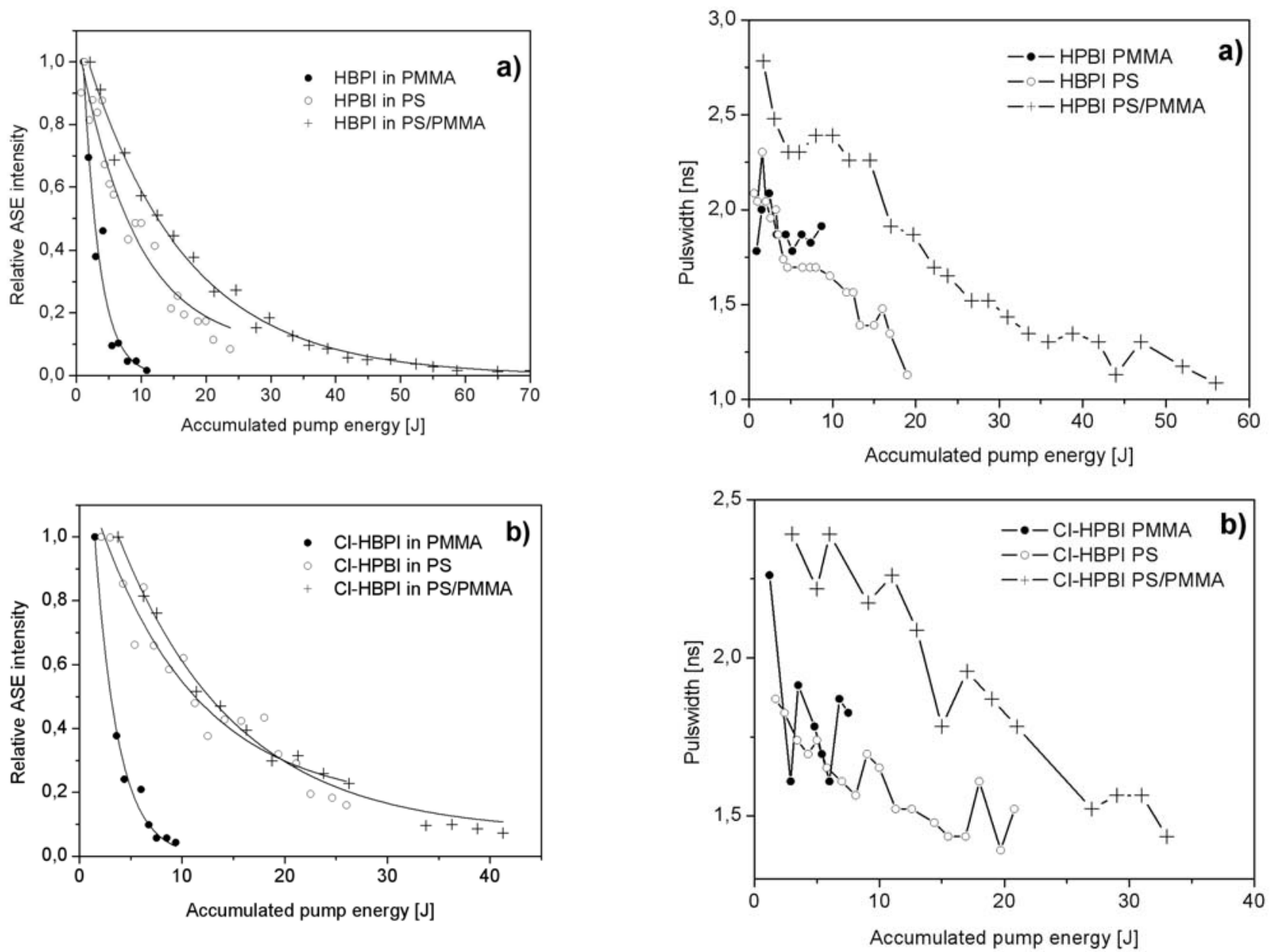

FIG. 3: Relative ASE output intensities vs. accumulated absorbed pump energy and first order exponential fit (solid lines), (a) HPBI, (b) $\mathrm{Cl}-\mathrm{HPBI}$.

ulation densities of all states $N_{i} / N_{0}$ constant for all $N_{0}$ as expected.

Figure 8 shows as an example the calculated and experimentally measured pulsewidth for HPBI in PS. For low concentrations of up to $4 \times 10^{17} \mathrm{~cm}^{-3}$ the behaviour the two curves is similar however the total values of the pulsewidth differ. Although neither intermolecular processes or interactions dye-polymer nor effects of the photo-degradation products to the laser process are introduced in this simple model, it basically reproduces the ASE pulse shortening observed in [7]. For higher population densities the ignored processes must be taken in consideration particularly with regard to the high dye concentrations in real dye laser applications.

\section{CONCLUSION}

The Amplified Spontaneous Emission of 2-(2'hydroxyphenyl)benzimidazole (HPBI) and 2-(2'-hydroxy5 '-chlorphenyl) benzimidazole (Cl-HPBI) incorporated in PMMA, PS and a PS/PMMA co-polymer was investigated.

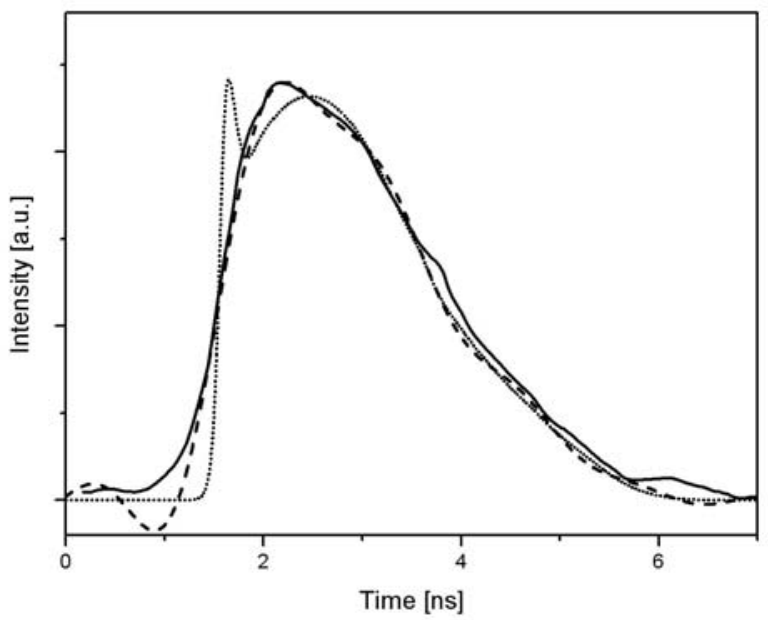

FIG. 5: Measured (dotted), calculated (dashed) and low pass filtered (solid) curves of the pulse of HPBI in PS/PMMA copolymer.

FIG. 4: Pulsewidth of the ASE vs. accumulated absorbed pump energy, (a) HPBI, (b) Cl-HPBI. 

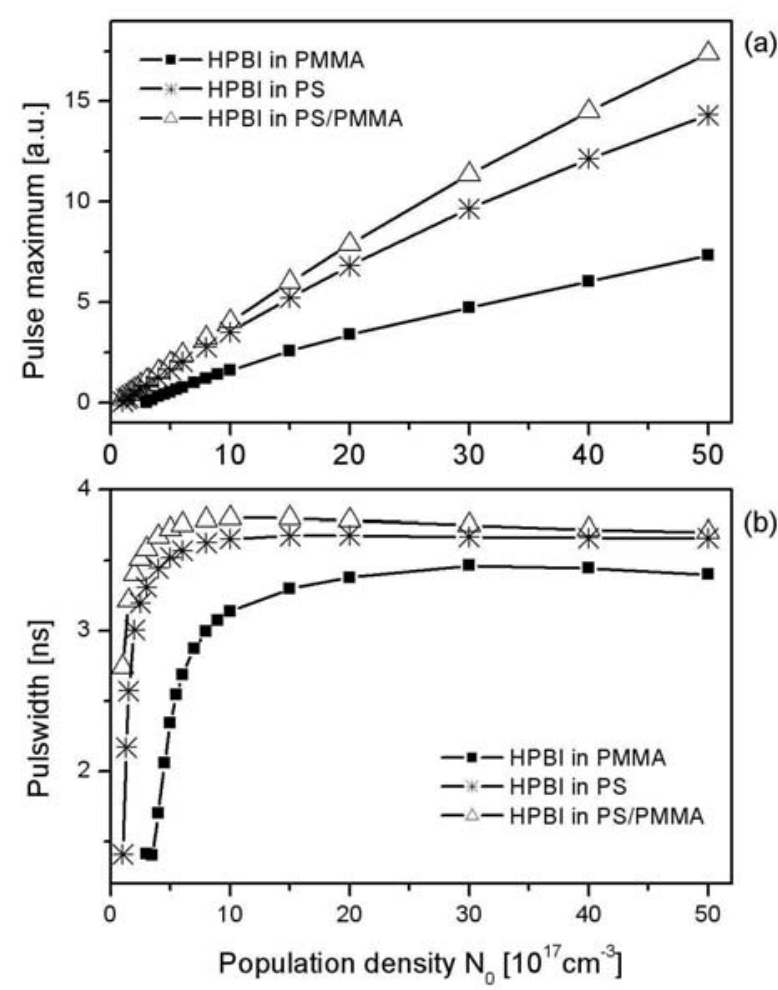

FIG. 6: Calculated ASE pulse maximum (a) and pulsewidth (b) for HPBI in PMMA, PS and PS/PMMA copolymer.

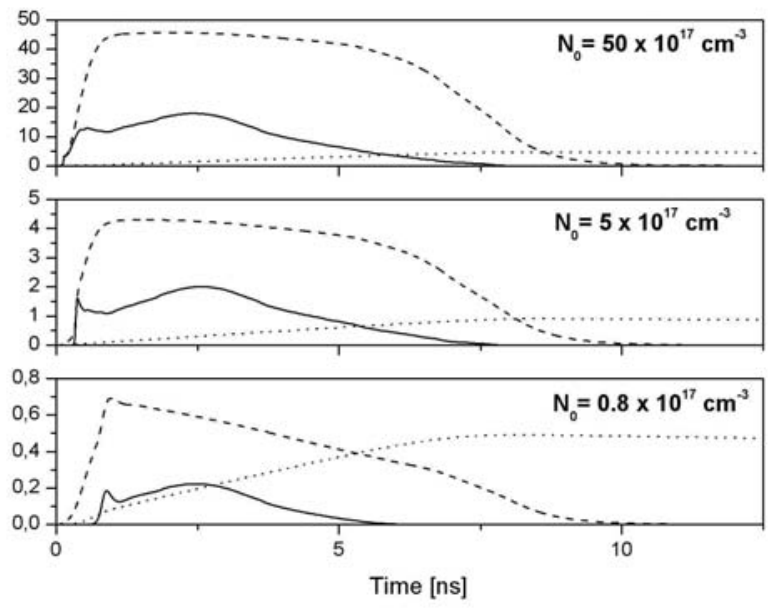

FIG. 7: Population densities of tautomer form fundamental singlet state $N_{0}^{\prime}$ (dashed line), normal form excited triplet state $N_{T}$ (dotted line) and ASE pulse intensity $I_{A S E}$ (solid line).
The co-polymer as host material shows highest emission efficiency and lowest photo degradation. To explain the degradation behaviour a rate equation system of four singlet and two triplet states was used. The model reproduce the ASE pulse, degradation process and pulse shortening in the different environments satisfactorily. The rise of triplet concentration in relation to the excited state singlet concentration

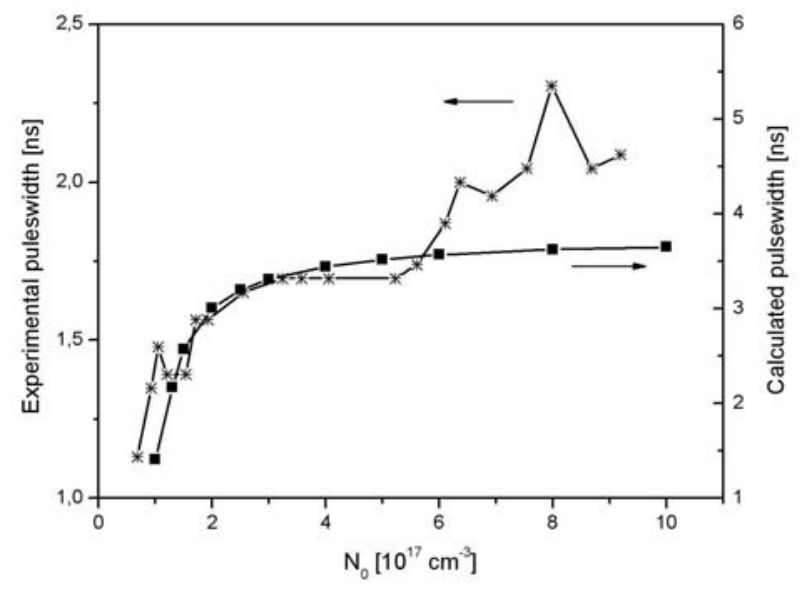

FIG. 8: Comparisons of experimental and calculated pulsewidth as function of $N_{0}$ for HPBI in PS.

within photo degradation process was supposed as the crucial process that leads to the pulse shortening observed in the experiments. The dye-polymer combination need further research to understand the degradation process to develop more photo stable systems based on proton-transfer dyes.

\section{Acknowledgments}

The authors would like to thank Prof. Dr. I. M. Brinn for the use of his facilities at LERT/UFRJ. This work was partially supported by the Conselho Nacional de Desenvolvimento Científico e Tecnológico (CNPq, 306212/2002-5), Coordenadoria de Aperfeiçoamento de Pessoal de Nível Superior (CAPES) and FAPERJ

The authors are indebted to Dr. S. G. Bertolotti from Universidad Nacional de Rio Cuarto, Argentina and Prof. Dr. F. Quina from Instituto de Química of the Universidade de São Paulo for the experimental facilities.
[1] S. Singh, V.R. Kanetkar, G. Sridhar, V. Muthuswamy, and K. Raja, J. of Luminescence 101, 285 (2003).

[2] M.L. Ferrer, A.U. Acuña, F. Amat-Guerri, A. Costela, J.M. Figuera, F. Florido, and R. Sastre, Applied Optics 33 (12), 2266 (1994).

[3] A. Costela, I. García-Moreno, R. Mallavia, F. Amat-Guerri, J.
Barroso, and R. Sastre, Optics Communications 152, 89 (1998).

[4] A. Costela, I. García-Moreno, J.M. Figuera, F. Amat-Guerri, J. Barroso, and R. Sastre, Optics Communications 130, 44 (1996).

[5] A. Costela, I. Garcia-Moreno, J.M. Figuera, F. Amat-Guerri, and R. Sastre, Applied Physics Letter 68, 593 (1996).

[6] A.U. Acuña, F. Amat-Guerri, A. Costela, A.Douhal, J.M. 
Figuera, F. Florido, and R. Sastre, Chemical Physics Letters $187(1,2), 98$ (1991).

[7] C.E. Fellows, C.C. Rodegheri, U. Täuber, K.H. Tsui, M.P.P de Castro, and C.E.M. Carvalho, Applied Physics B 78, 421 (2004).

[8] P. Chou, D. McMorrow, T.J. Aartsma, and M. Kasha, J. Phys. Chem. 88, 4596 (1984).
[9] C.E. Fellows, U. Täuber, C.C. Rodegheri, C.E.M. Carvalho, Diego F. Acevedo, Sonia G. Bertolotti, and Cesar Barbero, Opt. Mat. 27, 499 (2004).

[10] A. Costela, J.M. Muños, A. Douhal, J.M. Figuera, and A.U. Acuña, Appl. Phys. B 49, 545 (1989). 